\title{
Ein offener Wissensmarktplatz mit gesellschaftlicher
}

\section{Relevanz. Perspektiven der SLUB im digitalen Zeitalter ${ }^{1}$}

Zusammenfassung: In seiner Antrittsrede als Generaldirektor der Sächsischen Landesbibliothek Staats- und Universitätsbibliothek Dresden, beschreibt Achim Bonte Bibliotheken im digitalen Zeitalter als offene, diversifizierte Lernräume, die wesentlich von der Vielfalt kreativer Mitarbeiter und den Ideen der Benutzer leben. Auf dieser Grundlage sieht er die SLUB als lebendigen Kommunikationsort und Infrastruktureinrichtung, die mit ihren digitalen und nicht-textuellen Angeboten sowie ihren Diensten entlang des Forschungskreislaufs gut gerüstet in die Zukunft blickt. Schlüsselwörter: Offenheit, Vielfalt, Kommunikationsort, Digitalisierung

\section{An Open Marketplace of Knowledge with Social Relevance. Perspectives of the SLUB in the Digital Age}

Abstract: In his inaugural speech as chief executive of SLUB, Achim Bonte, describes libraries in the digital age as open and divers learning spaces, which gain from the diversity of creative employees and user-inspired ideas. Within this framework he sees the SLUB as a vital place for communication and digital information infrastructures. With its digital and non-textual offers and its services along the research cycle he considers the SLUB as well equipped for the future.

Keywords: Openness, diversity, communication, digitization

Verehrte Gäste aus nah und fern, an diesem besonderen Tag in meiner beruflichen Biografie stehe ich vor Ihnen mit ein wenig Stolz - und ziemlich viel Demut. - Stolz: Als ich vor gut zwanzig Jahren mit einer halben befristeten Stelle an der Universitätsbibliothek Heidelberg begonnen habe (ein herzlicher Gruß an alle unsere befristet Beschäftigten!), schien es keineswegs selbstverständlich, dass ich einst eine der größten deutschen Bibliotheken würde führen dürfen. Dies umso mehr, als schon der Weg bis nach Heidelberg vergleichsweise steinig war. Seit mehreren Generationen der erste in der Familie, der Abitur machen und studieren konnte, verdanke ich meine Entwicklung nicht zum geringsten dem Glück, in einem Land aufgewachsen zu sein, das Aufstieg durch Bildung gezielt

${ }^{1}$ Ansprache zur Amtseinführung als Generaldirektor der Sächsischen Landesbibliothek - Staats- und Universitätsbibliothek Dresden Dresden, 2. Juli 2018. Die Vortragsform wurde beibehalten und für den Druck nur geringfügig überarbeitet. 
Preprints der Zeitschrift BIBLIOTHEK - Forschung und Praxis, 2019, AR 3270 Bonte

Dies ist ein Preprint $(\boldsymbol{C})$ BY-NC-ND. Die endgültige Publikationsfassung erscheint beim Verlag De Gruyter unter https://www.degruyter.com/view/j/bfup

fördert. Wir alle wissen, dass Bildungsbenachteiligung auch in der Bundesrepublik trotzdem noch messbar ist. Gegenwärtig nehmen von 100 Akademikerkindern 74 ein Studium auf. Von 100 Nichtakademikerkindern studieren lediglich 21; und schon beim Erwerb der Hochschulreife zeigt sich die immer noch viel zu hohe soziale Selektivität des deutschen Bildungssystems. Bibliotheken können erheblich dazu beitragen, Bildungs- und Chancengerechtigkeit zu verbessern. Daran engagiert mitzuarbeiten, war für mich ursprünglich kein entscheidender Impuls für meine Berufswahl, betrachte ich heute aber als starke Motivation und innere Befriedigung.

Demut: „Nimm dich nicht so wichtig“, soll ein Engel dem vor 60 Jahren erwählten Johannes XXIII. einst eingegeben haben. Was selbst für einen großartigen Papst galt, sollte für uns alle erst recht gelten. Bezieht man die Vorläufereinrichtungen mit ein, ist die SLUB über 460 Jahre alt. Die zehn bis fünfzehn Jahre eines einzelnen Bibliotheksdirektors fallen dagegen nicht so sehr ins Gewicht, selbst wenn uns bisweilen der Eindruck beschleicht, ein Jahr sei heute so fordernd und ereignisreich wie etwa drei aus der vordigitalen Zeit. In dieselbe Richtung wirkt die Tatsache, dass ein Chef ohne sein Team nicht viel erreichen kann. Ich gehe noch weiter. Die Bibliothek ist nach meinem Verständnis nicht nur die Summe ihrer großen Tradition, ihrer Bestände und ihrer engagierten Mitarbeiterinnen und Mitarbeiter. Wesentlich sind auch das Wissen, die Kreativität und die Mitwirkungsbereitschaft ihrer Benutzerinnen und Benutzer. „Bibliotheken für die Menschen ist die alte Sicht, auf Bibliotheken zu schauen“, formuliert der amerikanische Bibliothekswissenschaftler Richard David Lankes. „Die neue Sicht sieht hingegen eine Bibliothek der Menschen. " Die lebendige Interaktion von Bibliothekarinnen und Bibliothekaren mit ihrem Umfeld, ihrer Community, deren Ideen und Engagement sind ein integraler Teil dessen, was eine Bibliothek jeweils ausmacht.

Keinerlei Anlass zu Überheblichkeit bietet schließlich auch der Sachverhalt, dass Bibliotheken wie alle Institutionen, die traditionell mit der Verteilung von Information, Unterhaltung und Wissen beschäftigt sind, seit Einführung des World Wide Web vor rund 25 Jahren unter einem besonders dramatischen, weiterhin wachsenden Veränderungsdruck stehen. Das Leistungsversprechen des mobilen Internets, digitale Inhalte und Dienste zu jeder Zeit, an jeder Stelle, situationsangepasst und personalisiert nutzen zu können, ist in zu vielen Bibliotheken in seiner revolutionären Bedeutung immer noch nicht ausreichend erfasst, mit jährlich 24 Millionen verkauften Smartphones in Deutschland aber gleichwohl Realität. Die gravierenden Folgen der Digitalisierung für die gesamte Informations- und Medienbranche lassen sich im privatwirtschaftlichen Sektor mühelos ablesen. Im Zeitungsangebot gingen in den letzten Jahren einst so bedeutende Blätter wie die „Frankfurter Rundschau“, der „Rheinische Merkur" oder die „Westfälische Rundschau“ verloren. Im Bereich der Publikumszeitschriften sind ganze Themengruppen ins Internet gewechselt. Im Börsenverein des Buchhandels ist die Mitgliederentwicklung stark rückläufig und fragt eine aktuelle Marktstudie „Buchkäufer - quo vadis?“. Bei den Videotheken werben Video-on-Demand-Dienste unverhohlen mit 
Preprints der Zeitschrift BIBLIOTHEK - Forschung und Praxis, 2019, AR 3270 Bonte

Dies ist ein Preprint $(\boldsymbol{C c})$ BY-NC-ND. Die endgültige Publikationsfassung erscheint beim Verlag De Gruyter unter https://www.degruyter.com/view/j/bfup

der Losung „Sparen Sie sich den Gang zur Videothek“. Im erbitterten Kampf um das wirtschaftliche Überleben und neue Marktchancen verschwimmt die vertraute Ordnung der Angebotssegmente und bieten Qualitätszeitungen zum Beispiel inzwischen auch Rechtsberatung und professionelle Faktenrecherchen oder Buchhandlungen Spiele und Geschenkartikel an.

Was ist eine Bibliothek im digitalen Zeitalter? Gewiss, auf hohem Abstraktionsniveau folgt sie unverändert dem klassischen Auftrag des Bildens und Vermittelns, sie verleiht auch noch Bücher und gewährt Zugang zu digitalen Objekten, sie muss aber mit ihren konkreten Räumen, Diensten und Hilfsmitteln zugleich noch viel mehr sein, wenn sie in Zukunft bestehen und sich hinreichend legitimieren will: Nicht nur Medienspeicher, sondern Wissensmarktplatz, nicht nur Abholstelle und Lesesaal, sondern diversifizierter Lernraum, nicht nur Arbeitsstätte für Bibliothekarinnen und Bibliothekare, sondern attraktiver Arbeitgeber für verschiedene weitere kreative Fachleute - und eben auch ein stets offenes Haus für unerwartete Ideen und Ergebnisbeiträge ihrer Benutzerinnen und Benutzer. Bibliotheken sind als öffentliche Einrichtungen bewusst nicht unmittelbar Marktzwängen ausgesetzt, folgen aber dennoch dem Prinzip von Angebot und Nachfrage. Wenn sie die Herausforderungen des Internetzeitalters längerfristig bestehen wollen, benötigen sie jede für sich genügend geschätzte Angebote der Bildung und Vermittlung, die ihnen anhaltend Zustimmung sichern.

Neben jeweils hochwertigen, geschätzten Dienstleistungen sind es die spezifischen Konditionen, die öffentlichen Bibliotheken im Zeitalter von Google \& Co. einen profilierten Platz und die Zukunft sichern. Bibliotheken sind konsequent offen für alle, niedrigschwellig und nicht-kommerziell, sie verkaufen keine Daten, achten auf die Seriosität der vermittelten Informationen und treten für offenen Austausch und offene Infrastrukturen ein. Gerade diese besonderen, für die Entwicklung unserer Gesellschaft eminent wichtigen Konditionen sind es, die wir gemeinsam wertschätzen und konsequent verteidigen sollten. Andererseits reichen sie alleine nicht aus, wenn Bibliotheken den Wettbewerb mit der Internet-Industrie tatsächlich bestehen wollen. Das zeigt das historische Beispiel des redlichen Tante-Emma-Ladens, dessen Schicksal einst viele bedauert haben, ohne darum ihre neuen Einkaufsgewohnheiten im System der preiswerten, wohlsortierten Supermärkte entscheidend zu ändern.

Die Frage, welche konkreten Dienstleistungen eine Bibliothek künftig erfolgreich bleiben lassen können, ist hoch flexibel und - je nach Auftrag und örtlichen Rahmenbedingungen - auch durchaus individuell zu beantworten: hinsichtlich der Tiefe und Materialität ihrer Informationsressourcen, der angestrebten Leistungsniveaus und Kenntnisschwerpunkte, der Reichweiten und Partnerschaften. Entsprechend werden Bibliotheken künftig weit weniger gleichförmig als in der Vergangenheit sein, wird die Bandbreite dessen, was „Bibliothek“ kennzeichnen und bedeuten kann, signifikant 
Preprints der Zeitschrift BIBLIOTHEK - Forschung und Praxis, 2019, AR 3270 Bonte

Dies ist ein Preprint $(\boldsymbol{C})$ BY-NC-ND. Die endgültige Publikationsfassung erscheint beim Verlag De Gruyter unter https://www.degruyter.com/view/j/bfup

zunehmen. Was also ist diese Bibliothek, was macht die SLUB zur SLUB? Diese Bibliothek ist zunächst gekennzeichnet durch einen besonders breiten gesetzlichen Auftrag. Als klassische Landesbibliothek sammelt und archiviert sie umfassend Veröffentlichungen über Sachsen sowie die in Sachsen erscheinenden ablieferungspflichtigen Publikationen. Als Bibliothek der Exzellenzuniversität TU Dresden trägt sie die Informationsversorgung einer forschungsstarken Volluniversität mit besonders breitem Fächerspektrum. Als größter Partner im Leistungsverbund der sächsischen Hochschulbibliotheken erfüllt sie wichtige Koordinierungs- und Dienstleistungsfunktionen im Freistaat, etwa im Rahmen des Landesdigitalisierungsprogramms, des digitalen Langzeitarchivs, der kooperativen Softwareentwicklung oder der konsortialen Erwerbung. Es sind diese umfangreichen Informationsinfrastrukturleistungen für ein Flächenland, die die SLUB klar von den üblichen Universitäts- und Landesbibliotheken unterscheiden. Wohl nur noch die Bayerische Staatsbibliothek engagiert sich in gleicher Intensität für die bibliothekarischen Belange eines Bundeslands. Und nach dem Muster der Bayerischen Staatsbibliothek bieten wir der sächsischen Staatsregierung aktuell an, uns strukturbildend und unterstützend auch für das derzeit noch dichte, angesichts der fortschreitenden Digitalisierung aber akut gefährdete Netz der kleinen und mittleren Öffentlichen Bibliotheken in Sachsen zu engagieren.

Von über 400 Öffentlichen Bibliotheken im Land werden aktuell 60\% nur neben- oder ehrenamtlich geleitet. Gut die Hälfte der Standorte verfügt über keinerlei digitale Arbeitsgeräte (elektronischer Katalog, Internetzugang u.ä.), drei Viertel bieten keine digitalen Medien zur Ausleihe an („Onleihe“). Zwei Drittel der Bibliotheken haben weniger als 20 Wochenstunden geöffnet, wobei sich die Öffnungszeiten in der Regel nach den Arbeitszeiten ihrer BetreuerInnen richten. Will heißen: nach 18 Uhr oder am Wochenende sind die Einrichtungen weit überwiegend geschlossen. Fragen Sie sich selbst, was solche Bibliotheken den Generationen $Y$ und Z, aber auch älteren berufstätigen Menschen wohl noch zu bieten haben. Dass ich hier vor allem Probleme von kleineren Gemeinden referiere, bestätigt eine neue, vom Rat für Kulturelle Bildung in Auftrag gegebene repräsentative Umfrage unter Bibliotheksleitungen. „Bibliotheken in Kleinstädten und Gemeinden beurteilen das Thema „Digitalisierung“ in ihrer Einrichtung vielfach anders als Bibliotheken in größeren Städten“, heißt es da. „Die unterschiedlichen Strukturmerkmale der Bibliotheken, wie Stadtgröße oder Zahl der Mitarbeitenden, spielen eine wesentliche Rolle. In den Bibliotheken der Großstädte ist die Digitalisierung nicht nur wesentlich weiter fortgeschritten als in den Gemeinden und Kleinstädten, sie verzeichnen zudem eine höhere Angebotsvielfalt und mehr digitale Serviceangebote.“

Falls es uns nicht gelingt, das traditionsreiche sächsische Bibliotheksnetz in seiner Breite angemessen voranzubringen, wird es innerhalb der nächsten zehn Jahren daher an immer mehr Stellen reißen. Falls es uns aber gelingt, können wir es hervorragend nutzen, um drängende Fragen der Entwicklung des ländlichen Raums und des sozialen Zusammenhalts produktiv anzugehen und Bibliotheken 
Preprints der Zeitschrift BIBLIOTHEK - Forschung und Praxis, 2019, AR 3270 Bonte

Dies ist ein Preprint $(\boldsymbol{C c})$ BY-NC-ND. Die endgültige Publikationsfassung erscheint beim Verlag De Gruyter unter https://www.degruyter.com/view/j/bfup

weiterhin als niedrigschwellige Zentren für Information, Unterhaltung und Bildung, als von vielen geschätzte, lebendige Orte für den notwendigen gesellschaftlichen Diskurs und sozialen Ausgleich zu behalten. Manche sächsische Gemeinde besitzt heute kaum noch eine Kneipe oder einen Laden, aber sie besitzt vielleicht noch einen Raum, der Menschen zusammenführen kann - sogar, ohne dass die das notgedrungen Geld kostet: die Bibliothek.

Um das Bibliotheksnetz in Sachsen erfolgreich zu modernisieren, benötigen wir unbedingt eine hoch leistungsfähige Landesfachstelle für Öffentliche Bibliotheken. Wir benötigen zentrale digitale Angebote wie die „Virtuelle Schulbibliothek Sachsen“. Vor allem aber benötigen wir den Willen der Kommunen, über die Gemeindegrenze hinauszudenken und verlässlich zusammenzuarbeiten. Nur auf diesem Weg werden wir uns flächendeckend die notwendigen Spezialkräfte (FachinformatikerInnen, MedienpädagogInnen, WebdesignerInnen) und Ausstattungsmerkmale (WLAN-Hotspots, kundenfreundliche Öffnungszeiten, attraktives Veranstaltungsangebot) leisten können, ohne die „Bibliothek“ in einem neuen, erweiterten Begriff schlichtweg nicht vorstellbar ist. Was für Bibliotheken gilt, gilt übrigens gleichermaßen für die kommunalen Archive und die ebenfalls rund 400 Museen in unserem Land. Es gibt kommunale Zweckverbände für Wasser, Abwasser, Müllentsorgung und wohl Manches andere. Lassen Sie uns auch mehr Zweckverbände für kulturelle Einrichtungen und Angebote schaffen.

Nur wer die Wurzeln kennt, weiß um die Kraft der Zweige. Die SLUB besitzt ein hochgradig der gemeinsamen Sache verpflichtetes und auch leidensfähiges Team. Aus der Zusammenführung von Universitätsbibliothek der TU und Sächsischer Landesbibliothek entstanden, mussten nach $1996 \mathrm{im}$ Zuge von Fusion und Neubauvorbereitung in kurzer Frist zahlreiche Mitarbeiterinnen und Mitarbeiter neue Aufgaben übernehmen, alle Betriebsabläufe vereinheitlicht und dezentrale Bibliotheken integriert werden. Zugleich galt es, empfindliche, historisch bedingte Bestandslücken an aktueller Literatur zu füllen und umfangreiche Bestände auszusondern oder neu zu signieren. Nach den harten Auseinandersetzungen um die Gründung der neuen Großbibliothek wurde dies noch begleitet von der heute längst widerlegten Skepsis der Anhängerschaft einer weiterhin eigenständigen „Sächsischen Landesbibliothek“, die bezweifelten, ob die Fusion auch tatsächlich das halten würde, was sich ihre Protagonisten davon versprachen. Der damalige Generaldirektor Jürgen Hering und seine Mannschaft haben diese Herausforderungen bravourös gemeistert und sich um die SLUB unschätzbare Verdienste erworben.

Als Thomas Bürger im Herbst 2003 den Staffelstab übernahm, bezog er sein Büro in einem neuen Gebäude mit einem umfangreichen neuen Dienstleistungsangebot und einem wohlgeordneten Bestand. Während die materielle Gründung der Bibliothek somit sichtbar zu einem gewissen Abschluss gekommen war, blieb noch als Aufgabe, die neue wissenschaftliche Großbibliothek aus 
Preprints der Zeitschrift BIBLIOTHEK - Forschung und Praxis, 2019, AR 3270 Bonte

Dies ist ein Preprint $(\boldsymbol{C c})$ BY-NC-ND. Die endgültige Publikationsfassung erscheint beim Verlag De Gruyter unter https://www.degruyter.com/view/j/bfup

„Deutsch-Fernost“ mit dem seltsamen langen Namen hinsichtlich Image, Kooperationsnetz sowie Innovations- und Drittmittelfähigkeit wieder dahin zu bringen, wo die traditionsreiche Sächsische Landesbibliothek in den 1920er Jahren bereits einmal gewesen war: auf die vorderen Plätze der Bibliotheks-Bundesliga. Trotz empfindlichen Personalabbaus hat die SLUB ihr Aufgabenfeld in den letzten fünfzehn Jahren in dreierlei Hinsicht wesentlich erweitert. Wie andere Bibliotheken hat sie erstens neben den klassischen Angebotsstrang der Papierbibliothek einen kraftvollen zweiten, rasch wachsenden Handlungsstrang digitaler Dienste gestellt. Dabei gibt es zwischen den beiden Aktionsfeldern vielfältige Beziehungen und eine gewisse Verdrängung zugunsten des Digitalen, werden die klassischen Leistungsangebote mit der Digitalisierung aber auf absehbare Zeit keineswegs restlos substituiert. In starker Erweiterung des traditionellen Auftrags der Verteilung von Information und Wissen hat die SLUB in enger Zusammenarbeit mit der Wissenschaft zweitens Dienste entlang des gesamten Forschungskreislaufs etabliert. In der SLUB erhalten Sie folglich nicht nur analoge und digitale Wissenskonserven, sondern auch Hilfe beim wissenschaftlichen Schreiben, beim Publizieren, bei der Wirkungsmessung, bei der Datenanalyse, beim Management von Forschungsdaten und manch anderem mehr. Schließlich hat sich die SLUB drittens prononciert der Welt des nichttextuell codierten Wissens geöffnet. Wissen war lange bevorzugt in Büchern und Texten gespeichert. Im Zuge der Digitalisierung und der damit erweiterten Möglichkeiten vollziehen sich Wissensaufbau, strukturierung und -vermittlung aber nicht nur mittels gedruckter oder digitaler Texte, sondern in wachsendem Maße auch mit nicht-textuellen Methoden. Während die Geisteswissenschaften mit möglichst umfassend verfügbaren, gut aufbereiteten digitalen Textarchiven arbeiten, vermissen die Ingenieur- und Naturwissenschaften in Bibliotheken vielfach fachadäquate Lernumgebungen. Technische Sachverhalte lassen sich in visualisierter Form und am realen Objekt oft leichter verstehen und diskutieren. Der viel beachtete SLUB Makerspace gibt mit einer rasch wachsenden Gerätevielfalt und kreativen Veranstaltungsformaten eine Antwort darauf und fördert zugleich unsere zentralen Anliegen, gemeinsam mit unseren Benutzerinnen und Benutzern Grenzen zu verschieben und neues Wissen zu schaffen. Nicht nur hier ist die SLUB so längst nicht mehr nur eine Dienstleistungs-, sondern eine forschungsnahe, explorative Infrastruktureinrichtung. Es liegt uns daher ebenfalls sehr am Herzen, dass der Staatsbetrieb SLUB im Interesse weiterer Kooperationsund Innovationschancen an geeigneten Hochschul- und Forschungsförderprogrammen des Landes in gleicher Weise teilhaben kann wie eine zentrale Informationseinrichtung der Hochschulen.

Wie geht es weiter mit der SLUB? Es ist heute nicht der Tag, ein fertiges Zukunftsprogramm vorzustellen. Jedoch sei gesagt, dass wir vor einigen Wochen mit fachkundiger Unterstützung einen Strategieprozess „SLUB 2025“ eingeleitet haben, der unter Berücksichtigung der sächsischen Hochschulentwicklungsplanung und relevanter überregionaler Papiere den mittelfristigen Weg der SLUB nach innen und außen noch deutlicher bestimmen soll. Einige mögliche Leitgedanken für das 
Preprints der Zeitschrift BIBLIOTHEK - Forschung und Praxis, 2019, AR 3270 Bonte

Dies ist ein Preprint $(\boldsymbol{C c})$ BY-NC-ND. Die endgültige Publikationsfassung erscheint beim Verlag De Gruyter unter https://www.degruyter.com/view/j/bfup

Strategiepapier habe ich in diesem Text bereits anklingen lassen. Nach meiner persönlichen

Überzeugung sollte die Bibliothek ihre Angebote künftig nicht nur streng kundenorientiert, sondern weitgehend partizipativ anlegen. Sie sollte trotz orts- und zeitunabhängiger Verfügbarkeit von Informationen und Wissen mit ihren physischen Gebäuden mehr denn je auch als öffentliches Studien- und Kommunikationszentrum, als leistungsfähiger und attraktiver dritter Ort zwischen Zuhause und Arbeitsplatz fungieren. Sie sollte gemäß unseres Claims „Wir führen Wissen“ Wissen in jeder Form organisieren und damit vermehrt auf die zunehmend bedeutsamen nicht-textuellen Zeichensysteme und Wissenswelten achten. Sie sollte mit aktivem Engagement für Openness auch eine prononciert gesellschaftspolitische Funktion erfüllen, die sichtbar über die Wissenschaftskommunikation hinausreicht; and last but noch least sollte die SLUB auch weiterhin produktiv aus ihrer reichen Vergangenheit und kulturellen Überlieferung schöpfen.

Um uns auch klar zu den Schattenseiten der Tradition zu bekennen, sitzen Sie seit heute - wie vielleicht manche bemerkt haben werden - nicht mehr im „Vortragssaal“, sondern im „KlempererSaal“ der SLUB. Der Klemperer-Saal trägt der Tatsache Rechnung, dass hier inzwischen längst nicht nur Vorträge, sondern zum Beispiel auch Musik- und Filmdarbietungen, Diskussionen und Debatten, World-Cafés und andere Workshop-Methoden Platz finden. Vor allem aber erinnert er an mehrere verdiente Persönlichkeiten, die zu Dresden und der SLUB in besonderer Beziehung standen. Victor Klemperer hatte ab 1920 bis zu seiner Amtsenthebung durch die Nationalsozialisten 1935 den Lehrstuhl für Romanistik an der Technischen Universität Dresden inne. Eine der vielen Repressalien während der Diktatur war ein Benutzungsverbot der Sächsischen Landesbibliothek. Seit 1977 konnte die SLUB die Tagebücher Klemperers sowie weitere Dokumente der Familie übernehmen. Die Tagebücher 1933-1945 wurden in viele Sprachen übersetzt, dienten als Filmstoff und sind heute ein Standardwerk im Geschichts- und Deutschunterricht. Nicht näher verwandt mit dem Romanisten war der Dresdner Bankier Victor Klemperer von Klemenau dessen 1938 beschlagnahmte Büchersammlung, darunter 549 wertvolle Inkunabeln, in die Bibliothek gelangte. Aus den Ausweichdepots kamen nach Kriegsende lediglich 12 Inkunabeln zurück. 1991 wurde der verbliebene Rest der Sammlung Klemperer an seine Erben restituiert. Victor Klemperers Vater Gustav, Direktor der Dresdner Bank und ebenfalls ein bedeutender Sammler, war Förderer und Ehrensenator der Technischen Universität Dresden. Das Beispiel der Klemperers erinnert uns daran, was Dresden einst an geistiger und kultureller Substanz ohne Not preisgegeben hat.

Lassen Sie mich abschließend danken: Herrn Bürger für zwanzig höchst arbeitsreiche, verantwortungsbeladene Jahre im Dienste der SLUB. Meinen Vorrednern für Ihre anerkennenden Worte, meiner Familie, meinen Freunden und Weggefährten für Ihre vielfältige, unschätzbare Unterstützung sowie den Kolleginnen und Kollegen des SLUB-Chors für die musikalische Einlage und auch Ihnen, verehrte Festgäste, für Ihr so zahlreiches Erscheinen am heutigen Tag. - Bleiben Sie mir 
Preprints der Zeitschrift BIBLIOTHEK - Forschung und Praxis, 2019, AR 3270 Bonte Dies ist ein Preprint $(c c)$ EY-NC-ND. Die endgültige Publikationsfassung erscheint beim Verlag De Gruyter unter https://www.degruyter.com/view///bfup

und bleiben Sie der SLUB auch künftig gewogen - für eine sichere, selbstbestimmte und kraftvolle Entwicklung dieses Hauses in einer offenen und freien Wissensgesellschaft.

Herzlichen Dank!

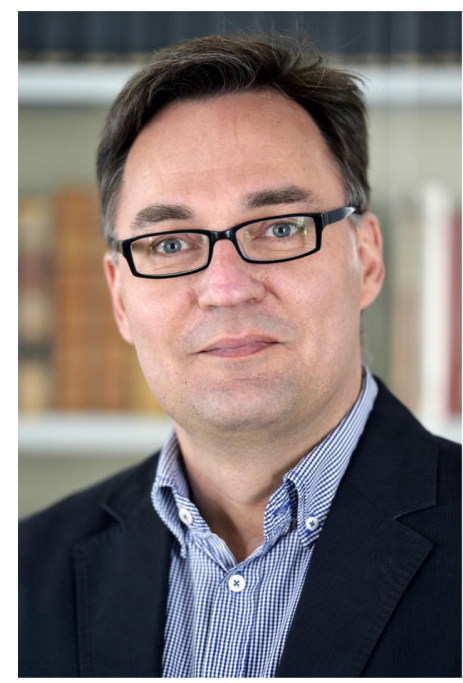

Dr. Achim Bonte

Generaldirektor

Sächsische Landesbibliothek - Staats- und Universitätsbibliothek Dresden (SLUB)

D-01054 Dresden

Besucheradresse: Zellescher Weg 18

achim.bonte@slub-dresden.de 